\title{
Analysis of Four-Wing Fractional-Order Qi System and Its Application in Secure Communication
}

\author{
Hongyan Jia ${ }^{1,}$, Qinghe Wang 2, b \\ ${ }^{1}$ Department of Automation, Tianjin University of Science and Technology, Tianjin 300222, China \\ ${ }^{2}$ Department of Automation, Tianjin University of Science and Technology, Tianjin 300222, China \\ aemail: jiahy@tust.edu.cn, bemail: lifei_1997@126.com
}

Keywords: fraction-order; lyapunov exponent diagrams; bifurcation diagrams; circuit implementation; secure communication.

Abstract. In this paper, a newly reported four-wing fractional-order Qi system is discussed and analyzed. Its dynamic characteristic is observed through investigating the Lyapunov exponent diagrams, bifurcation diagrams, and phase orbits diagrams when the order is as low as 2.7. Then, based on the four-wing fractional-order Qi system, an analog circuit for illustrating the application in secure communication is designed and analyzed. The experimental result shows that its application for secure communication is practical.

\section{Introduction}

Although fractional calculus has a history of more than 300 years [1], the applications of fractional calculus to physics and engineer have only become a hot topic in recent years. Since the chaos phenomenon has been found in large number of fractional-order system, the dynamic characteristics of the fractional system has become a research hotspot. Some fractional-order chaotic systems have been proposed and researched, such as the fractional-order Lorenz system [2], the fractional-order Chen system [3,4,5,6], the fractional-order of Lü system [7].

As a newly reported chaotic system, Qi system has aroused extensive research of experts and scholars. It was first discovered and proposed by Qi [8] et al. It is found that its dynamic characteristics are very complex, and with different parameters, the system will show different chaotic phenomenon. So far, many research achievements of Qi system have been put forward. Such as, based on topologic horseshoe map theory, Jia et al. proved the existence of four-wing chaotic attractor in Qi system [9]; On the basis of the Qi system, Xue et al. proposed and analyzed a novel four-wing hyper-chaotic system [10]. Utilizing topologic map theory, Guo et al. analyzed the existence of topologic horseshoe in a four-wing fractional-order Qi system [11], and so on.

With the rapid development of digital and network technology, the significance of information security has been important in the past few decades. Intrinsic properties of chaotic systems such as sensitivity to initial conditions, and a wide spectrum bandwidth of system signal, it makes the chaotic system have an outstanding advantages and important application prospect in the field of secure communication. Especially the fractional order systems are more common in the field of secure communication. So it has important theoretical significance and practical value to investigate the application of fractional order chaotic system in secure communication field.

In this paper, a fractional-order Qi system is firstly discussed and analyzed, and the Lyapunov exponent diagrams and bifurcation diagrams of the fractional-order Qi system are also given to verify its chaotic characteristic. On the basis of synchronous control, an analog circuit is designed to study its application for secure communication. The result from experiment shows that its application for secure communication is practical.

\section{Four-wing Fractional-order Qi system}

The four-wing fractional-order Qi system can be written as 


$$
\left\{\begin{array}{l}
\frac{d^{\alpha} x_{1}}{d t^{\alpha}}=a\left(y_{1}-x_{1}\right)+e y_{1} z_{1} \\
\frac{d^{\alpha^{\alpha} y_{1}}}{d t^{\alpha}}=c x_{1}+d y_{1}-x_{1} z_{1} \\
\frac{d^{\alpha} z_{1}}{d t^{\alpha}}=-b z_{1}+x_{1} y_{1}
\end{array}\right.
$$

Where $0<\alpha<1$ refer to the fractional order, system parameters a,b,c,d,e $\in \mathrm{R}$. This paper mainly discusses the condition of the fractional order $\alpha=0.9$. It is found that the system has a four-wing chaotic attractor whena $=14, \mathrm{~b}=43, \mathrm{c}=-1, \mathrm{~d}=16$, and $\mathrm{e}=4$, as shown in Fig. 1 . Generally speaking, the positive Lyapunov exponent is an important feature to show chaotic behavior of the system. Therefore, the Lyapunov exponent diagrams of the fractional-order system and bifurcation diagrams are discussed in this paper. When selectingb $=43, \mathrm{c}=-1, \mathrm{~d}=16, \mathrm{e}=4$, and varying a, the Lyapunov exponent diagrams and bifurcation diagram of system are shown in Fig.2 (a) and (b), respectively. From the Fig.2, chaos behavior may exist in the system when a $\in[6,22]$. Here, the cases of varying b, varying c, varying d, and varying e are no longer discussed. By the analysis, we can conclude that chaotic behaviors exist in the system (1) when the order is as low as 2.7. In this paper, the four-wing chaotic attractors will be adopted to investigate its applications in secure communication.

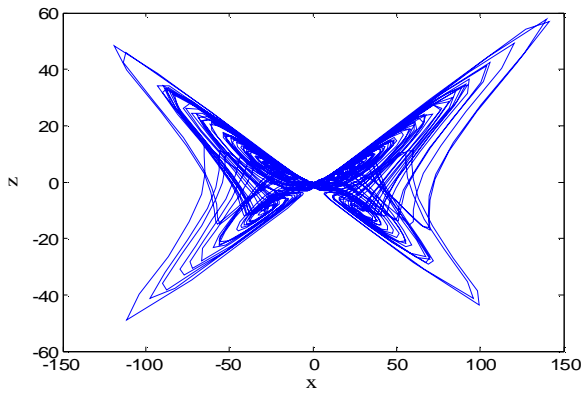

(a)

Fig.1 Chaotic attractors (a) $\mathrm{x}_{1}-\mathrm{z}_{1}$

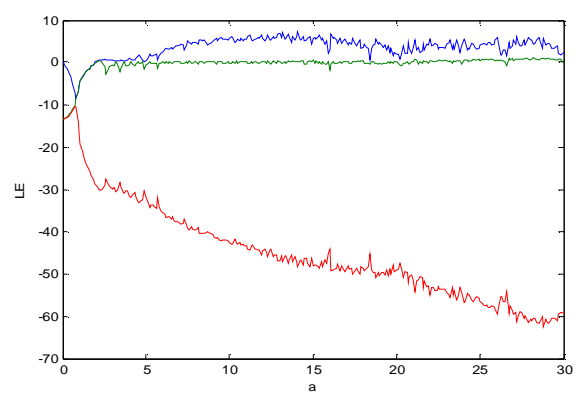

(a)

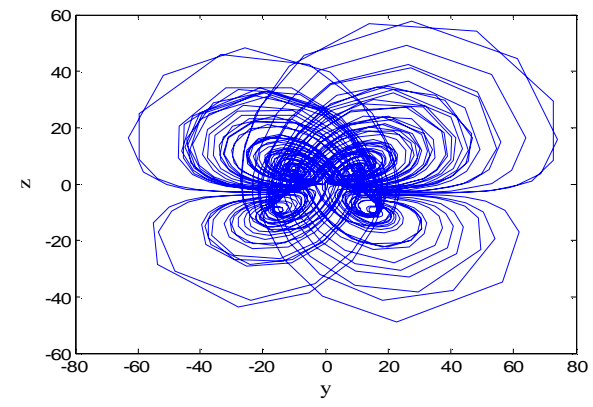

(b)

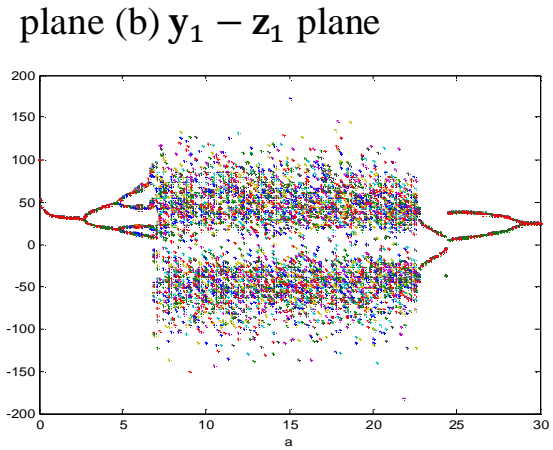

(b)

Fig.2 Lyapunov exponent diagrams and Bifurcation diagram withb $=43, \mathrm{c}=-1, \mathrm{~d}=16, \mathrm{e}=$ 4 and varying a.

\section{Synchronization design and Circuit implementation}

It is well known that chaos synchronization is the foundation of the application of chaotic system in secure communication. So the synchronization of four-wing fractional-order Qi system is firstly investigated and will be adopted to implement its application in secure communication. Here, the system (1) can be acted as a driving system, and the corresponding response system is written 


$$
\left\{\begin{array}{l}
\frac{\mathrm{d}^{\alpha} \mathrm{x}_{2}}{\mathrm{dt} \mathrm{t}^{\alpha}}=\mathrm{a}\left(\mathrm{y}_{2}-\mathrm{x}_{2}\right)+\mathrm{ey}_{2} \mathrm{z}_{2}+\mathrm{u}_{1} \\
\frac{\mathrm{d}^{\alpha} \mathrm{y}_{2}}{\mathrm{dt^{ \alpha }}}=\mathrm{cx}_{2}+\mathrm{dy}_{2}-\mathrm{x}_{2} \mathrm{z}_{2}+\mathrm{u}_{2} \\
\frac{\mathrm{d}^{\alpha} \mathrm{z}_{2}}{\mathrm{dt^{ \alpha }}}=-\mathrm{bz}_{2}+\mathrm{x}_{2} \mathrm{y}_{2}+\mathrm{u}_{3}
\end{array}\right.
$$

Where $\mathrm{u}_{1}, \mathrm{u}_{2}$, and $\mathrm{u}_{3}$ are the control input to realize the synchronization of system (1) and system (2). According to the linear feedback synchronization theory and the stability theory of fractional order linear systems, selecting

$$
\left[\begin{array}{l}
u_{1} \\
u_{2} \\
u_{3}
\end{array}\right]=\left[\begin{array}{c}
e_{1} z_{1}-e y_{2} z_{2} \\
-x_{1} z_{1}+x_{2} z_{2} \\
x_{1} y_{1}-x_{2} y_{2}
\end{array}\right]+\left[\begin{array}{ccc}
0 & a & 0 \\
c & d+1 & 0 \\
0 & 0 & 0
\end{array}\right]\left[\begin{array}{l}
x_{2}-x_{1} \\
y_{2}-y_{1} \\
z_{2}-z_{1}
\end{array}\right]
$$

the system (2) will be synchronized with the system (1).

Then we design an analog circuit for synchronization control to verify the correctness of the theoretical analysis, as shown in Fig.3. In the circuit, LF347N is selected as the amplifier, AD633 (output gain is 0.1) is selected as the multiplier, and $R_{1}=R_{3}=R_{18}=R_{20}=714 \Omega$, $\mathrm{R}_{2}=\mathrm{R}_{19}=12.5 \Omega, \mathrm{R}_{4}=\mathrm{R}_{10}=\mathrm{R}_{15}=\mathrm{R}_{21}=\mathrm{R}_{27}=\mathrm{R}_{32}=1.55 \mathrm{M} \Omega, \mathrm{R}_{5}=\mathrm{R}_{11}=\mathrm{R}_{16}=\mathrm{R}_{22}=\mathrm{R}_{28}=\mathrm{R}_{33}=62 \mathrm{M} \Omega, \mathrm{R}_{6}=$ $\mathrm{R}_{12}=\mathrm{R}_{17}=\mathrm{R}_{23}=\mathrm{R}_{29}=\mathrm{R}_{34}=2.5 \mathrm{k} \Omega, \mathrm{R}_{7}=\mathrm{R}_{24}=\mathrm{R}_{35}=10 \mathrm{k} \Omega, \mathrm{R}_{8}=\mathrm{R}_{14}=\mathrm{R}_{25}=\mathrm{R}_{31}=50 \Omega, \mathrm{R}_{9}=625 \Omega$, $\mathrm{R}_{13}=\mathrm{R}_{30}=233 \Omega, \mathrm{R}_{28}=588 \Omega, \mathrm{C}_{1}=\mathrm{C}_{4}=\mathrm{C}_{7}=\mathrm{C}_{10}=\mathrm{C}_{13}=\mathrm{C}_{16}=0.7 \mu \mathrm{F}, \mathrm{C}_{2}=\mathrm{C}_{5}=\mathrm{C}_{8}=\mathrm{C}_{11}=\mathrm{C}_{14}=\mathrm{C}_{17}=0.52 \mu \mathrm{F}$, $\mathrm{C}_{3}=\mathrm{C}_{6}=\mathrm{C}_{9}=\mathrm{C}_{12}=\mathrm{C}_{15}=\mathrm{C}_{18}=1.1 \mu \mathrm{F}$. The experimental results are only given the synchronous phase trajectory and timing sequence diagram on the $\mathrm{x}_{1}-\mathrm{x}_{2}$, as shown in Fig.4, the rest is no longer listed. From the result, we can observe that the two chaotic systems are synchronized.
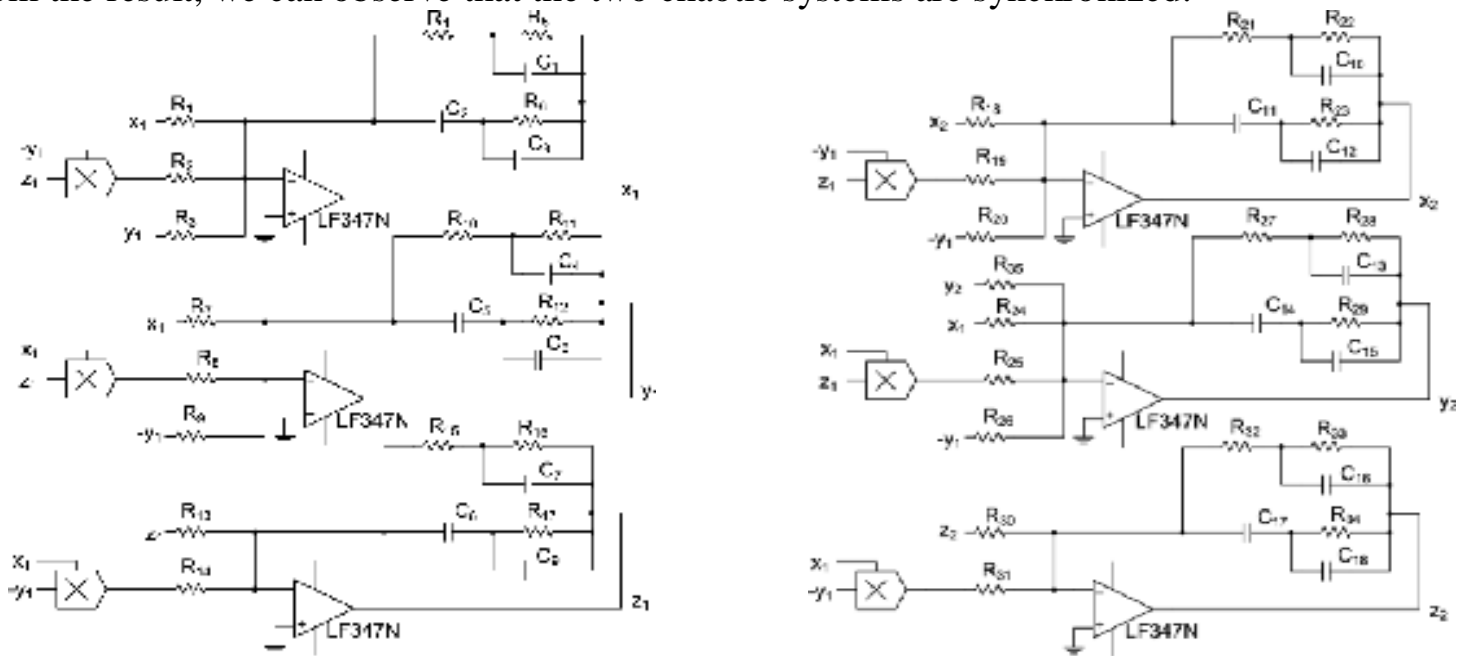

Fig.3 Circuit implementation of synchronization between system (1) and system (2)

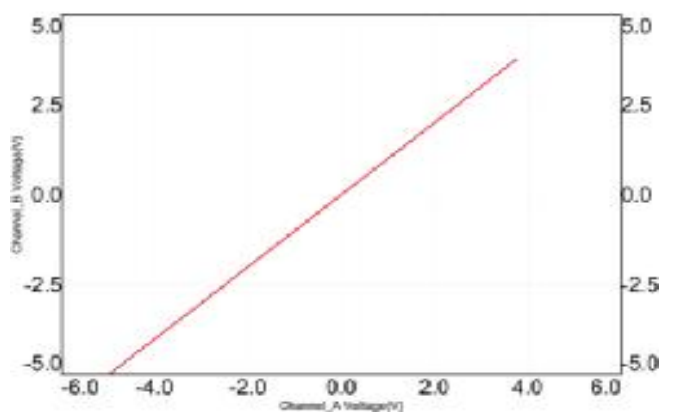

(a) Time series of $x_{1}-x_{2}$

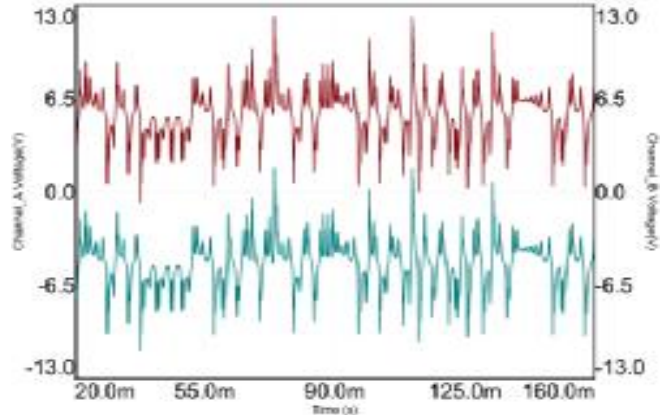

(b) Phase synchronization of $x_{1}-x_{2}$

Fig.4 Experimental result of synchronization 


\section{Secure Communication based on Chaotic Synchronization}

In this paper, on the basis of the above analog circuit of synchronization, an analog circuit for secure communication is designed, as shown in Fig.5. Here, we select the state variable $\mathrm{x}_{1}$ as the driving carrier signal, and a sine signal as original signal to be encrypted. Meanwhile the corresponding response signal $\mathrm{x}_{2}$ is selected as the decrypt signal. The experimental results are shown in Fig.6, and the decryption signal can be observed to be exactly like the original signal. That is to say, the four-wing fractional-order Qi system can be used in secure communication.

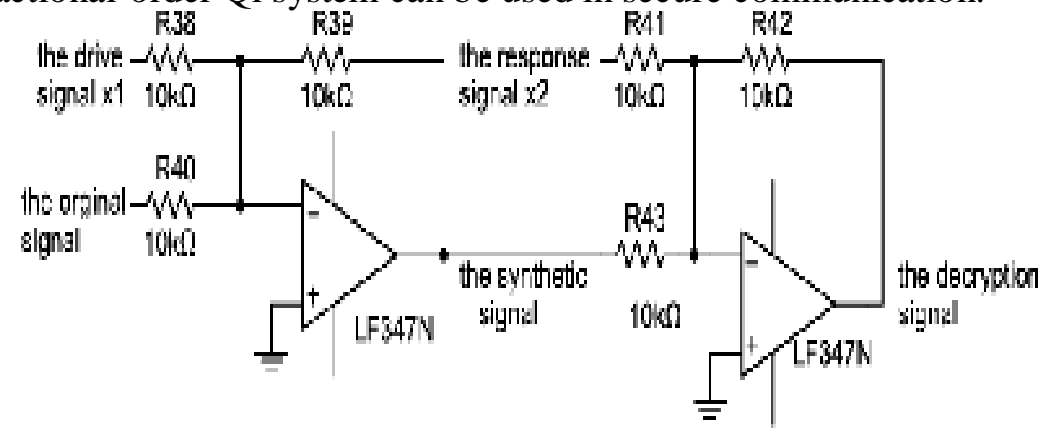

Fig.5 Analog circuit on secure communication

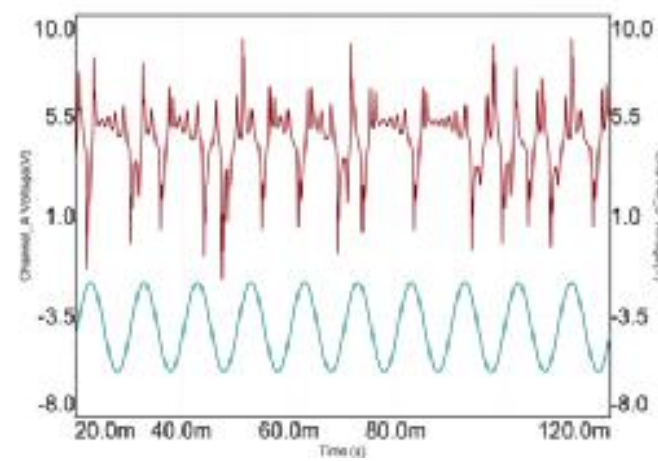

(a) The drive signal-The original signal

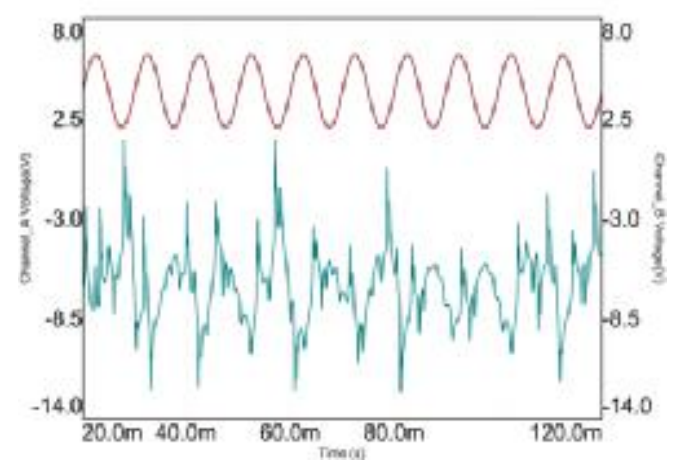

(c) The original signal-The synthetic signal

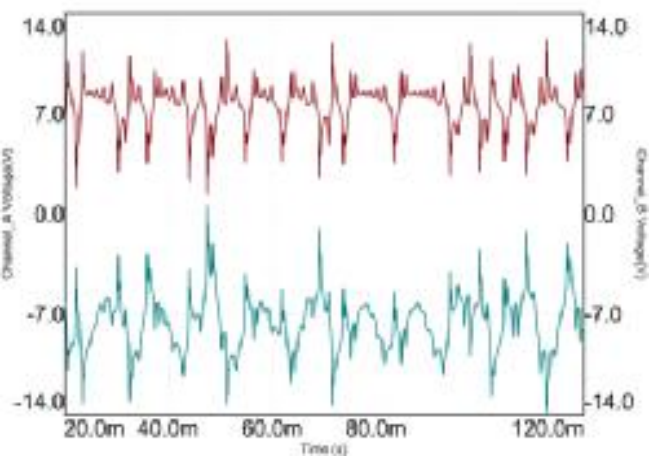

(b) The drive signal-The synthetic signal

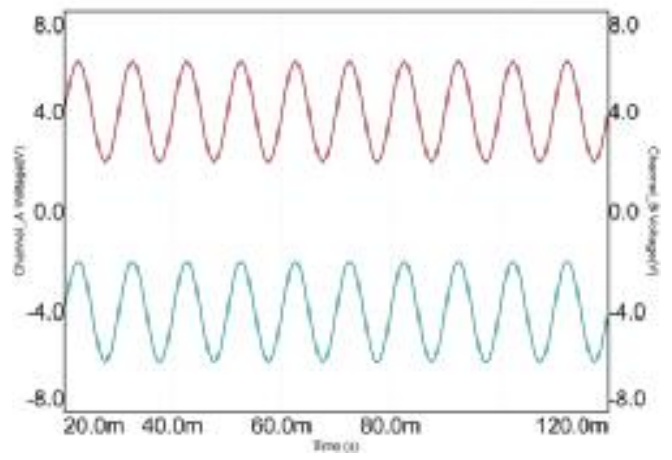

(d) The original signal-The decryption signal

Fig.6 The experimental results of secure communication

\section{Conclusions}

This paper firstly discusses and analyzes a four-wing fractional-order Qi system. Its chaotic characteristic can be observed by numerical simulation when the order is as low as 2.9. Then the existence of chaos is verified through the Lyapunov exponent diagrams, bifurcation diagrams, and phase orbits diagrams. A chaotic synchronization of two four-wing fractional-order chaotic systems with identical structure is realized. Meanwhile, an analog circuit is also designed to implement the synchronization control. At last, a kind of chaotic masking technology is used to investigate the chaotic secure communication based on the synchronization control, and the result from experiment 
shows that the encryption method is practical. This also provides a new model and technology support for the study on chaotic encryption.

\section{Acknowledgements}

This work was supported in part by the Young Scientists Fund of the National Natural Science Foundation of China (Grant No. 11202148), the Natural Science Foundation of China(Grant No. 61174094).

\section{References}

[1] G. Ilia, and G. Elena: Phys. Rev. Lett Vol. 91 (2003), p. 034101

[2] Yongguang Yu, Hanxiong Li, Sha Wang, and Junzhi Yu: Chaos, Solitons and Fractals Vol. 42 (2009), p. 1181

[3] Junguo Lu: Physica A Vol. 35 (2006), p. 107

[4] Junguo Lu, and Guanrong Chen: Chaos, Solitons and Fractals Vol. 27 (2006), p. 685

[5] Changpin Li, and Guojun Peng: Chaos, Solitons and Fractals Vol. 22 (2004), p. 443

[6] Chunguang Li, and Guanrong Chen: Chaos, Solitons and Fractals Vol. 22 (2004), p. 549

[7] Junguo Lu: Physics Letters A Vol. 354 (2006), p. 305

[8] Guoyuan Qi, Guanrong Chen, M.A. van Wyk, and B.J. van Wyk: Chaos, Solitons and Fractals Vol. 38 (2008), p.705

[9] Hongyan Jia, Zengqiang Chen, and Fei Ye: Acta Phys. Sin Vol. 60 (2011), p. 010203

[10]Wei Xue, Guoyuan Qi, Jingjing Mu, Hongyan Jia, and Yanling Guo: Chin. Phys. B Vol. 22 (2013), p. 080504

[11] Yanling Guo, and Guoyuan Qi: Journal of Applied Analysis and Computation Vol. 5 (2015), p. 168 